\title{
Electron Holographic Tomography of Mean Free Path Lengths at the nm-scale
}

\author{
Axel Lubk ${ }^{1}$, Daniel Wolf ${ }^{1}$, Falk Röder ${ }^{1}$ and Hannes Lichte ${ }^{1}$ \\ 1. Triebenberg Laboratory, TU Dresden, Dresden, Germany.
}

In off-axis Electron Holography a Möllenstedt biprism is introduced slightly above an intermediate image plane (image coordinates $\mathbf{r}_{\perp}$ ) to generate an interference pattern ("hologram") in the latter with the following cosinoidal intensity distribution

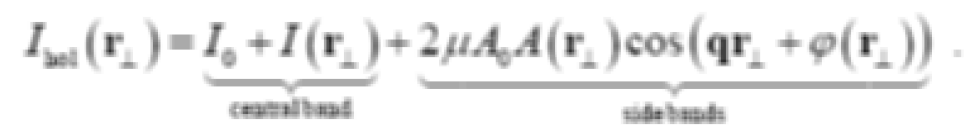

Here, $I_{0}, I, \mu, A_{0}, A, \mathbf{q}$ and $\varphi$ are the reference intensity, conventional image intensity, contrast damping factor due to camera MTF and partial coherence, reference amplitude, reconstructed amplitude, carrier frequency and reconstructed phase. The technique has been successfully applied to measure various electrostatic (and magnetostatic) potential characteristics [1] exploiting the relationship

$$
\varphi\left(\mathbf{r}_{\perp}\right)=C_{E} \int V(\mathbf{r}) \mathrm{d} z
$$

between reconstructed phase and potential largely valid for out-of-zone-axis conditions. Eq. (2) represents a Radon transformation when performed over a $\pi$-range of tilt angles, facilitating a 3D potential reconstruction from such a series [2]. A similar projection transformation may be obtained for the concomitantly recorded reconstructed image intensity $I$ and amplitude $A$

$$
\ln \frac{A\left(\mathrm{r}_{\perp}\right)}{A_{0}}=\frac{1}{2} \int \lambda_{A}^{-1}(\mathbf{r}) \mathrm{d} z \text { and } \ln \frac{I\left(\mathrm{r}_{\perp}\right)}{I_{0}}=\int \lambda_{I}^{-1}(\mathbf{r}) \mathrm{d} z
$$

with corresponding attenuation coefficients $\lambda^{-1}$.

In this contribution we will show how to extract elastic and inelastic mean free path lengths (MFPL) from the above attenuation coefficients correcting a serious misinterpretation preventing quantitative analysis in the past. Based on these considerations we will show how to tomographically reconstruct (in)elastic MFPLs in a fully quantitative manner thereby exploiting the full 3D information content of a holographic tilt series. That includes adaptions and simplifications of the standard phase reconstruction scheme like dedicated normalization and dynamic scattering correction and the omission of phase distortion correction by means of empty holograms.

We will demonstrate the MFPL reconstruction at a GaAs- $\mathrm{Al}_{1 / 3} \mathrm{Ga}_{2 / 3} \mathrm{As}$ core shell nanowire. Here, the GaAs nanowire has been grown by low pressure metal-organic vapor phase epitaxy (MOVPE) method using colloidal Au nanoparticles (NPs) as metal catalysts. GaAs/AlGaAs core-shell nanowires were then fabricated by epitaxial overgrowth. The tilt series was recorded at a Cs-corrected FEI TITAN TEM at $300 \mathrm{kV}$. Amongst other features its reconstructed potential reveals the core-shell structure as well a potential slope of unknown origin at towards the Au tip (Fig. 1). The reconstructed elastic MFPL data (Fig. 1) also reveals the core-shell structure as well as a chemical composition variation from AlAs to 
GaAs in the tapered region thereby facilitating an unambiguous interpretation of the above noted potential slope in terms of chemical composition change. The inelastic MFPL (Fig. 1) on the other hand only vaguely hinds the existence of the core-shell which is a result of the strong delocalization of the dominating bulk plasmon excitation in the inelastic MFPL.

Both elastic and inelastic MFPL agree very well with theoretic predictions. The precision of the MFPL is generally lower compared to the potential data due to the lower SNR in both reconstructed amplitude and intensity.

[1] Midgley, P. A. \& Dunin-Borkowski, R. E. Nat Mater, 2009, 8, 271-280.

[2] Wolf, D.; Lubk, A.; Röder, F. \& Lichte, H., Current Opinion in Solid State and Materials Science , 2013, 17, 126-134.

[3] McCartney, M. R. \& Gajdardziska-Josifovska, M, Ultramicroscopy, 1994, 53, 283-289. [4] The authors acknowledge financial support from the European Union under the Seventh Framework Programme under a contract for an Integrated Infrastructure Initiative. Reference 312483 - ESTEEM2.

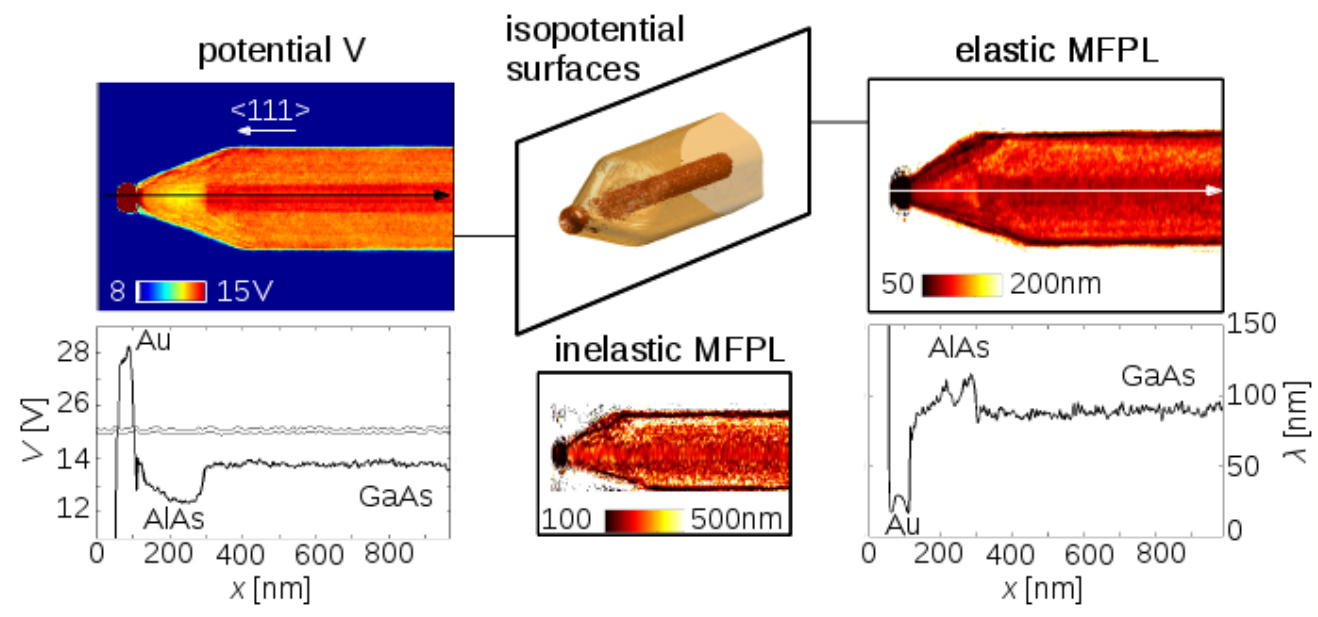

Figure 1. Short compendium of 3D data obtained from holographic tomographic reconstruction of GaAs-AlGaAs core-shell nanowire. Left column: Reconstructed potential cross-section and corresponding linescan exhibiting core-shell structure as well as potential slope in the tapered region. Right column: Reconstructed elastic mean-free-path-length (MFPL) cross-section and corresponding linescan also revealing core-shell structure and similar slope in tapered region. Based on the potential and the elastic MFPL a chemical composition change from AlAs to GaAs in the tapered region is identified. The inelastic MFPL data does not reveal a sharp core-shell structure. Additionally, one observes a strong damping (small MFPLs) at the boundary of the nanowire due to large angle Fresnel scattering. 